\title{
HYDRODYNAMIC MODELING OF ACCRETION SHOCKS ON A STAR WITH RADIATIVE TRANSPORT AND A CHROMOSPHERIC MODEL
}

\author{
J.-P. Chièze ${ }^{1}$, L. de Sá ${ }^{1,2}$ and C. Stehlé ${ }^{2}$
}

\begin{abstract}
Accretion flows on the surface of a star is modeled using a high resolution hydrodynamic 1D ALE code (ASTROLABE) coupled to radiative transfer and line cooling, along with a model for the acoustic heating of the chromospheric plasma.
\end{abstract}

\section{Introduction}

One dimensional hydrodynamic models (Koldoba et al. 2008; Sacco et al. 2008; Sacco et al. 2010) have confirmed the radiative shock origin of the soft X-ray observations of CTTSs (Testa et al. 2004; Robrade \& Schmitt 2007), showing periodic variations due to radiative instabilities. Furthermore, two dimensional MHD models (Orlando et al. 2010) have stressed the dependence of the dynamics and of the stability of accretion shocks upon the plasma parameter $\beta$. However, in these simulations, cooling of the shock heated plasma is entirely attributed to "on the spot" line cooling, discarding radiation transfer. This may be a crude approximation in the relatively dense part of the cooling flow, which penetrates the chromosphere. The treatment of the structure of the chromosphere itself is also simplified in these simulations, although the depth at which occurs the main accretion shock, and thus its observability, depends on the pressure profile of the chromosphere. We report here on a first attempt to include both radiative transfer and a model of stellar chromosphere heating in hydrodynamics simulations of accretion flows on stellar surfaces.

\section{Physical assumptions and model}

The radiatively driven evolution of an accretion flow impacting the dynamical structure of the chromosphere of a star is modelized by solving the 1D equations

\footnotetext{
1 CEA/DSM/IRFU/SAp-AIM, CEA Saclay, Orme des Merisiers, 91191 Gif-sur-Yvette, France

2 Observatoire de Paris/LERMA, UMR 8112, Université Pierre et Marie Curie, CNRS, 5 place J. Janssen, 92195 Meudon Cedex, France
} 
of hydrodynamics coupled to radiation, encompassing the optically thick to thin plasma regimes, relevant respectively to the upper photosphere and the shocked material.

\subsection{Code specificities}

The 1-D ASTROLABE code is a fully implicit Adaptive Lagrangian Eulerian (ALE) code with a fixed number of mesh points, which can move independently of the fluid velocity, to adapt the resolution to the flow properties (Dorfi \& Drury 1987; Lesaffre et al. 2004). The accretion column is treated with a height dependent section: it may be for instance cylindrical, conical or exponential. The ionization fraction is calculated according to the Saha equation, which is here modified in order to encompass photoionization and Lyman $\alpha$ radiation trapping (Brown 1973). However, this is a crude approximation, since coronal equilibrium, a balance between collisional ionization and radiative \& dielectronic recombination, is relevant for the present physical conditions. This improvement is currently being included in our code. The variation of the adiabatic exponent is taken into account by including the ionization energy of atoms in the expression of the internal energy of the plasma.

Radiative transfer is described by the two time dependent equations for radiation energy $\left(E_{r}\right)$ and momentum densities $\left(\mathcal{M}_{r}\right)$, which are written in the comoving frame (Mihalas \& Mihalas 1984; Lowrie et al. 2001). The necessary closure relation is obtained using the so-called $M 1$ prescription, where the expression of the Eddington factor $f_{E d d}=P_{r} E_{r}$ is obtained by maximizing the entropy of the radiation field (Levermore 1996; Dubroca \& Feugeas 1999). In regions where departures from thermodynamical equilibrium are weak, the terms which couple these two equations for $E_{r}$ and $\mathcal{M}_{r}$ are:

$$
\left\{\begin{array}{l}
S_{E_{r}}=\kappa_{P} \rho c\left(a T^{4}-E_{r}\right) \\
S_{\mathcal{M}_{r}}=-\kappa_{R} \rho F_{r}
\end{array}\right.
$$

where, we have assumed Planck and Rosseland grey opacities, $\kappa_{P}$ and $\kappa_{R}$. However, in the upper, hot, low density regions, the optically thin plasma is in coronal equilibrium. This is the case in the shocked material of the accretion flow. In this case, the source term for radiative energy is $S_{E_{r}}=n_{e} n_{H} \Lambda(T)$, where $n_{e}$ and $n_{H}$ are respectively the volume densities of electrons and of hydrogen nuclei, and $\Lambda(T)$ is the plasma cooling function. The source term of the radiation momentum vanishes: $S_{\mathcal{M}_{r}} \approx 0$. In the present work we use the cooling function of Kirienko (1993).

\subsection{A generic model for chromosphere}

Models of stellar chromosphere generally provide temperature and density profiles in hydrostatic equilibrium, adjusted to fit the observed chromospheric spectrum. An ad hoc heating function ( $E_{H}$, using the notation of Peres et al. 1982) may 
be derived as a function of the radius, in order to maintain the equilibrium of chromosphere. The energy input $E_{H}$ mimics actual energy deposition by sound waves, Alfvén waves and electronic conduction, which, since the pioneering work of Biermann (1946) and Schwarzschild (1948), are thought to heat the chromospheric layers. However, this procedure cannot be unambiguously adopted to determine the depth of the stagnation point of the accretion flow in the chromosphere, which depends crucially on the structure of the (unsteady) pressure profile of the upper stellar atmosphere. Our first approach has been to include the acoustic, dynamical, heating of the chromosphere in the global accretion model, leaving to further $3 \mathrm{D}$ calculations chromospheric heating by hydromagnetic waves. The aim here is to get a self-consistent, fully hydro-radiative description of the chromospheric structure, impacted by the accretion flow.

Thus, we have investigated the behavior of acoustic waves on the structure of the outer layers of a star (we choose the Sun for comparisons with theoretical models and observations, see e.g. Rammacher \& Ulmschneider 1992, Kalkofen 2007). Mechanical energy is supplied at the base of the simulation domain (at $\tau \geq 1$ ), in the form of a monochromatic sinusoidal motion of the first (Lagrangian) interface. Heating of the corona is not taken into account, since the later is readily crushed by the accretion flow. Figure 1 shows the formation of traveling shocks, induced by acoustic waves. Heating of the chromosphere is the result of the time-averaged temperature structure above the photosphere.

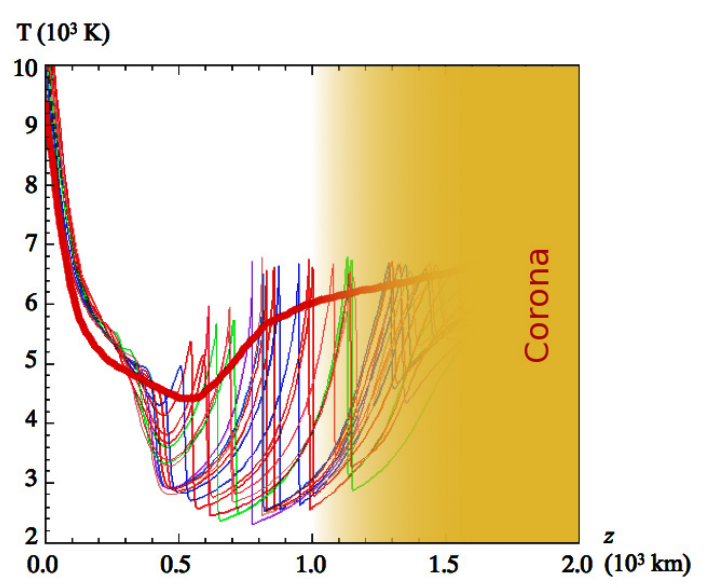

Fig. 1. Formation and propagation of shock waves induced by sound waves with initial energy flux (at $z=0 \mathrm{~km}$ ) of $10^{8} \mathrm{erg} \mathrm{cm}^{-2} \mathrm{~s}^{-1}$ and a period of $60 \mathrm{~s}$. The thin lines are successive snapshots of the temperature structure of the chromosphere and the thick line represents the mean temperature of the solar chromosphere (Th. Lanz, private communication). Above $500 \mathrm{~km}$, acoustic waves degenerate into shocks, which strength is governed by the balance between steepening in the pressure gradient and dissipation.

\section{First results}

The main features of accretion flows, especially their periodic behavior, are conserved when they interact with a dynamic chromosphere modelized as described previously. This is illustrated by Figure 2, which presents a complete cycle, with 
the formation of the X-ray emitting reverse shock, followed by the cooling of the shocked material. Cooling drives the formation of a second shock in the upper chromosphere and the crushing of the whole structure onto the chromosphere. After complete cooling, a new reverse shock is driven in the infalling material. The oscillation period is about $300 \mathrm{~s}$. However, the structure of the flow at the base of the accretion column is significantly modified both by radiative transfer and by the dynamics of the chromosphere. It turns out that a crucial issue is the treatment of the transition between the collision dominated plasma (inner regions) and the non equilibrium external regions, where coronal equilibrium prevails.
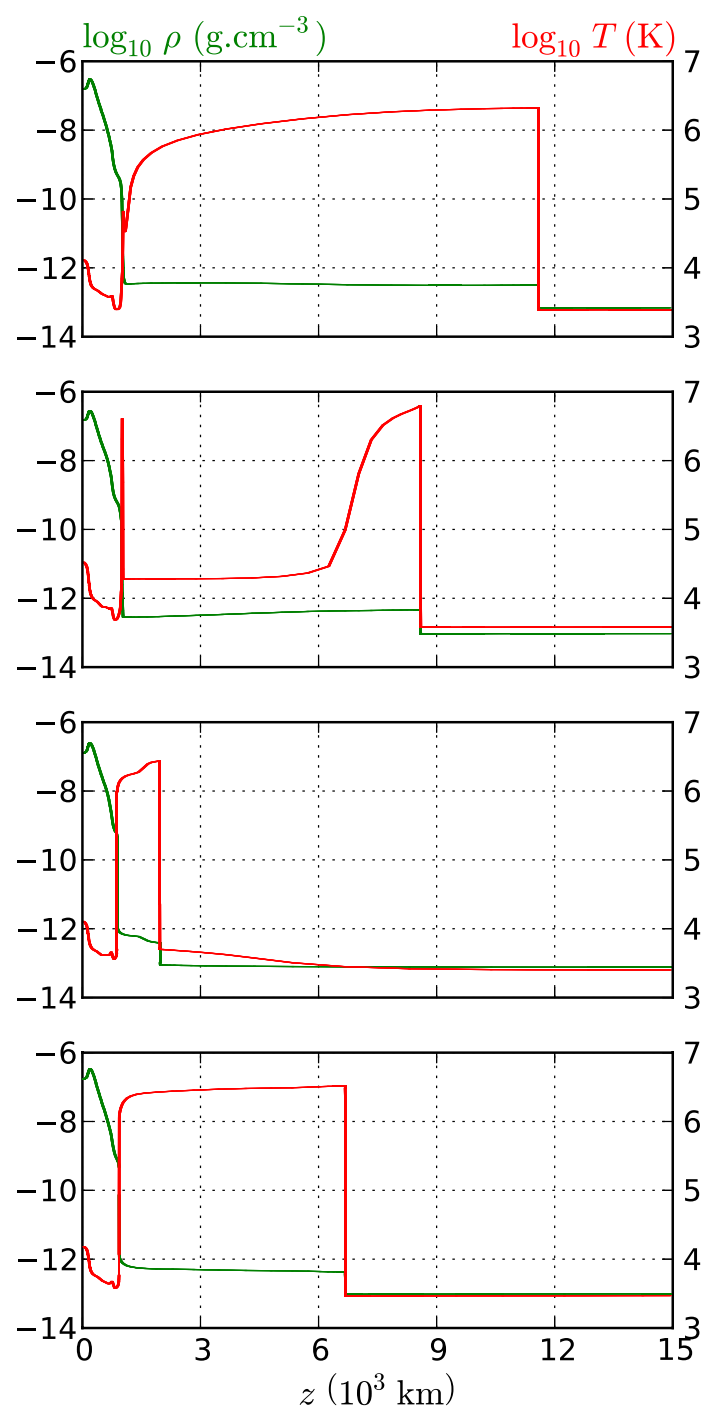

Fig. 2. Shock cycle showing 4 snapshots of the density $\rho$ in $\mathrm{g} / \mathrm{cm}^{3}$ (green line) and temperature $T$ in $\mathrm{K}$ (red line). The gas is infalling from the right to the left on a dynamical chromosphere, heated as in Figure 1. Snapshots are equally spaced by $60 \mathrm{~s}$. From top to bottom: a reverse shock forms and propagates outwards. The shocked material cools down under quasi isochoric conditions; a strong inwardly directed pressure gradient forms, which launches a second shock into the chromosphere. The whole structure is finally crushed on the chromosphere, and a new reverse shock forms. The period of this cyclic evolution is about 300 s. Cycle $n^{\circ} 20$ is shown here. 


\section{Conclusion}

The numerical model outlined in this paper includes for the first time the treatment of the radiative transfer in the flow and a self-consistent model of the stellar chromosphere, in order to precisely characterize the thermodynamical and radiative properties of the densest part of accretion column, which is the strongest XUV emitter. In a next step, a grid of models will be calculated in order to post-process the detailed spectra emerging from these structures.

This work was supported by French ANR (grant 08-BLAN-0263-07), PICS4343 of CNRS, University Pierre et Marie Curie, Observatoire de Paris and CEA Saclay. We thank Thierry Lanz and Ivan Hubeny for their helpful support.

\section{References}

Biermann, L., 1946, Naturwissenschaften, 33, 118

Brown, J.C., 1973, Sol. Phys., 29, 421

Dorfi, E.A., Drury, L. O'C, 1987, JCoPh, 69, 175

Dubroca, B., \& Feugeas, J.-L., 1999, C.R. Acad. Sci., 329, 915

Feautrier, P., 1964, SAO Spec. Rep., 167, 80

Kalkofen, W., 2007, ApJ, 671, 2154

Kirienko, A., 1993, Astron. Lett., 19, 11

Koldoba, A.V., Ustyogova, G.V., Romanova, M.M., \& Lovelace, R.V.E., 2008, MNRAS, 388,357

Lesaffre, P., Chièze, J.-P., Cabrit, S., \& Pineau des Forêts, G., 2004, A\&A, 424, 147

Levermore, D., 1996, J. Stat. Phys., 83, 1021

Lowrie, R.B., Mihalas, D., \& Morel, J.E., 2001, J. Quant. Spec. Radiat. Transf., 69, 291

Mihalas, D., \& Mihalas, B.W., 1984, Foundations of Radiation Hydrodynamics (New York: Oxford University Press)

Orlando, S., Sacco, G.G., Agiroffi, C., et al., 2010, A\&A, 510, A71

Peres, G.,Serio, S., Vaiana, G.S., \& Rosner, R., 1982, ApJ, 252, 791

Rammacher, W., \& Ulmschneider, P., 1992, A\&A, 253, 586

Robrade, J., \& Schmitt, J.H.M.M., 2007, A\&A, 473, 229

Sacco, G.G., Agiroffi, C., Orlando, S., et al., 2008, A\&A, 491, L17

Sacco, G.G., Orlando, S., Argiroffi, C., et al., 2010, A\&A, 522, A55

Schwarzschild, M., 1948, ApJ, 107, 1

Testa, P., Drake, J.J., \& Peres, G., 2004, ApJ, 617, 508 